\title{
VISUALIGATION TECHNIQUE FOR ROBOTIC COMPONENT CONNECTION SYSTEMS
}

\author{
B. L. Atkina, S. O. Popo-Ola ${ }^{\mathrm{b}}$, R. D. Wing ${ }^{\mathrm{b}}$

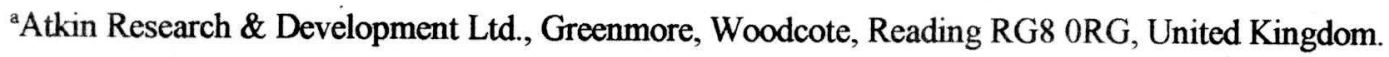 \\ bDepartment of Civil Engineering, Imperial College of Science, Technology and Medicine, South \\ Kensington, London SW7 2BU, United Kingdom.
}

\begin{abstract}
As the construction process moves towards an increasingly automated scenario, the design of fixing systems which can be easily handed by robotic devices becomes an essential factor in the eventual viability of the whole system. This project has studied many common and many uncommon fixing methods, in a survey covering all areas of engineering industry. Some of the methods are already in use in factory automation systems; however, for use with robotic and automation equipment on construction sites a more detailed and innovative approach to assessing the suitability of connectors is required.

The paper reports on the use of a mechanical modelling software package which has been integrated into a visualisation system to provide a realistic simulation of the engineering performance of the connector under simulated site conditions. The software permits application of quantified forces and restraints to the mechanical model. This technique has been used as a preliminary stage in the design and assessment of connectors for robotic assembly, and has thereby avoided the necessity of prototype testing numerous different design possibilities.

As a demonstration of the method, examples of various feasible connector types for exterior wall cladding with robotic assembly tools are given, these being a rationalised set of fixing systems to satisfy a wide range of sizes, material types, and anchor provisions. It is suggested that this addition to the scope of prefabricated components will generally improve the safety of assembly, assurance of attachment, and provide labour and financial savings to the cladding industry.
\end{abstract}

\subsection{INTRODUCTION}

The assembly and fixing of cladding panels is generally labour intensive and involves potentially dangerous tasks. Heavy panels are manhandled into position using a combination 
of cranes, scaffolds and ladders, and the nature of current fixing systems requires operatives to assemble brackets and bolts in awkward positions ${ }^{1}$. The process lends itself to automation, but requires substantial investment in purpose-built manipulators and innovative fixing systems. Operators would benefit from removal of much of the physical effort required and safer conditions; constructors would see an improvement in reliability and quality of the product.

\subsection{BACKGROUND}

Several fixing methods have been identified and grouped into classes of usage by different sectors of the engineering industry ${ }^{2}$. The processes which are always carried out when fixing or jointing components can be generalised into a sequential list:

Preparation of components; Assembly of components; Mating of assembly to fixing device (or vice versa); Initiation of fixing process; Withdrawal of the machine when fixing is complete; Post-fixing inspection, finishing and adjustment.

All these operations are present in one form or another in every fixing type investigated. Some fixing methods require more of one particular process than others; some combine several of these processes into a single stage, but the general principle is the same and all the stages mentioned above must be present

Most of the common fixing methods can be automated to a high degree in a factory setting, and well-established principles are used to redesign components and fixings to ensure that the automated process is reliable, efficient, and cost-effective. Applying these principles within the unstructured and inhospitable conditions which prevail on most building sites, however, requires a far more detailed and innovative approach than for the factory situation.

EPSRC funded researchers at Imperial College and University of Reading are investigating the potential of various novel forms of jointing for new fixing systems; these include highperformance adhesives, robotic rivetting, and component interlock techniques as used in the electrical industry. The aim of the work is to obtain a rationalised set of fixing systems for cladding that will satisfy a wide range of material types, will adapt to the increasing use of onsite automation and off-site prefabrication, will generally improve the safety of this aspect of building construction, and provide real savings to the cladding industry.

\subsection{CLADDING, and VISUALISATION AS A DESIGN TOOL}

Traditionally, external walls were constructed as load bearing elements, using basic materials such as masonry and brick ${ }^{3}$. as shown in Figure 1 . Today, non-load bearing external walls are commonplace with loads transferred to the ground by means of a frame ${ }^{4}$. Because external walls no longer need to be load bearing, it is possible to clad buildings in a variety of modern materials using a modular carrier matrix for support ${ }^{5}$. The components of such modular systems are manufactured using modern production techniques, but on-site assembly of the

13th ISARC $\quad-692-$ 


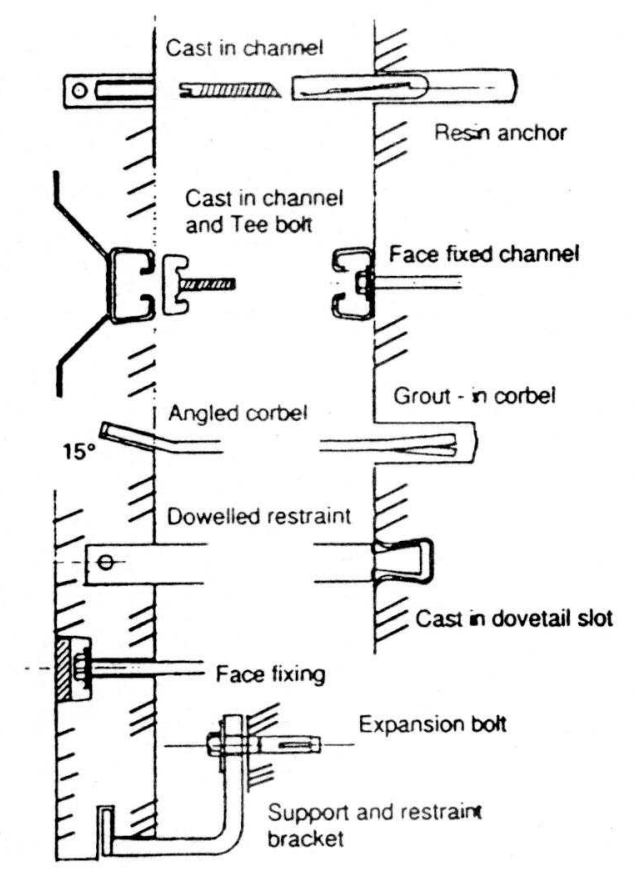

Figure 1 Common fixings for cladding. system lacks the automation attributes it deserves. The authors believe that redesign of the assembly and fixing processes for automation devices is a necessary next step, and that the use of recent developments in visualisation for engineering design will hasten the evolution of this part of the process.

Visualisation is now finding serious application in many branches of engineering, notably in the aircraft and automobile sectors, where the building of real prototypes can now be minimised. The technique can be applied to a process such as wall cladding at two levels: (i) the macro level, where the complete building model is visualised together with the assembly of the cladding components and visualisations of the site equipment to be used, and (ii) the micro level, where the detail of processes

such as (in this case) the connectors and their assembly can be modelled and studied.

The usefulness of such visualisations extends beyond the design process; it facilitates explanation of the processes to be used to contractors and technicians involved in the actual work, and presents a model for use as a working standard.

\subsection{MECHANICAL MODELLING}

Modelling of the geometry of processes such as connector assembly at micro level can be easily achieved using one of the excellent object modelling software packages available today. This approach is too limited for effective mechanism design, and a package that allows true modelling of mechanisms including applied forces was sought. A virtual mechanical/realistic object simulation package created by the University of Stuttgart for general mechanism design work (Animation Editor for Realistic Object Motion -X-AERO) proved effective for our purposes, and was adopted as the underlying modeller for this study.

It should be made clear that the object of this work was to develop the tools for designing component assembly systems rather than the detailed design of the systems themselves. Mechanical simulation models to demonstrate the techniques as applied to cladding fixings have been developed using XAERO. The package allows the creation of virtual environments from scenes created from simple 3-Dimensional geometrical objects (sphere, cylinder, box, point and plane). Objects can be linked to each other by a variety of methods (rod, spring, damper, and joint). 
Realistic object movements are achieved by a simulation procedure, where forces are applied to the objects in addition to gravity, friction and air resistance. Collisions between objects are simulated using either the "penalty method" or the "analytical method" 6.

\subsection{CREATION OF VIRTUAL CONNECTORS}

The scene editor (typical screen display shown in Figure 2) is used to create the image; it allows the user to enter solid (3D) objects, forces, reactions, and connections, using four views to display the model. By selecting different materials, the user can influence physical properties such as density or coefficient of friction and

elasticity. The user can accelerate or induce rotation of bodies in a defined direction using forces.

After creating the virtual

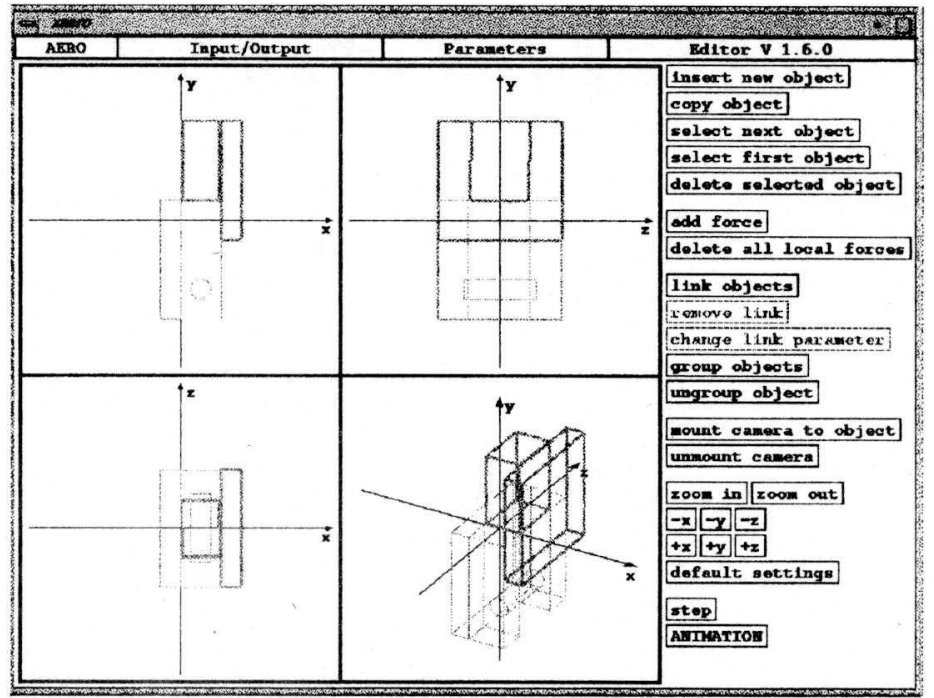

Figure 2. XAERO input screen editor image with all the necessary forces and connections between members in place, the data are then saved in a file. On compiling this file a sequence file is generated which contains parameters for step-by-step synchronization points (sync points) in the animation. Since any number of sync points can be declared, the sequence can be divided up into steps as small as required. The playback of these sequences using the animation feature provided with XAERO allows the generation of new output files for every image frame which can then be used for ray tracing. This process is carried out using another software package, POV (persistence of vision), to create output files compatible with the MPEG movie encoding and decoding standard.

Several models connectors have been developed using the AERO package described above. The output of the modelling process is normally presented in the form of an MPEG video, but for the presentation of this work on the printed page, wire and photorealistic frame sequence animation figures are used; clearly, the force and gravity attributes are not visible in the processed video stills. A number of the demonstration models of cladding connectors developed using the process described above are presented in the following section in order of increasing complexity.

\subsection{CONNECTOR MODELS - over}



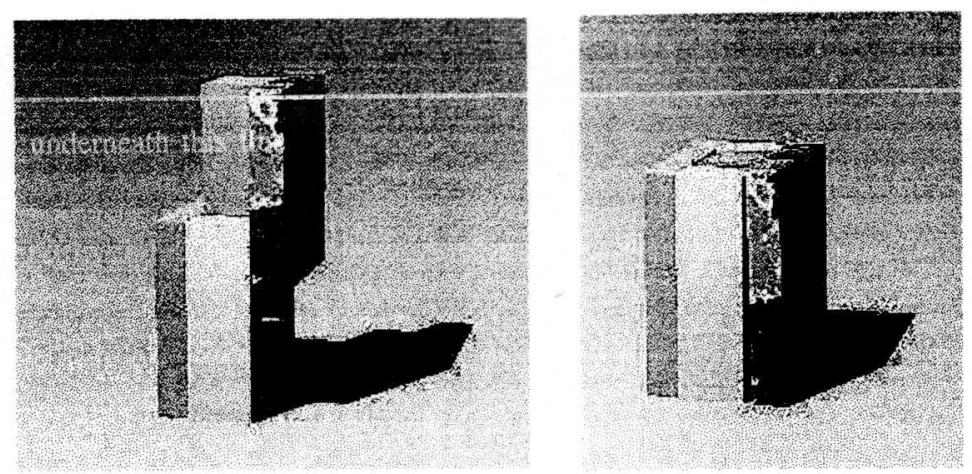

GROOVE:

Figures 3.1 (a) and (b)

The model 'groove' uses the concept of simple tongue and groove technique, whereby a connecting groove element is attached to the structure, and a corresponding tongue is attached to the back of the cladding panel. The cladding is then positioned in such a way that the connecting tongue can slide into the connecting groove element. The final locking is then provided by the weight of the cladding panel, which keeps the tongue and groove mechanism in place as shown in the figures above.
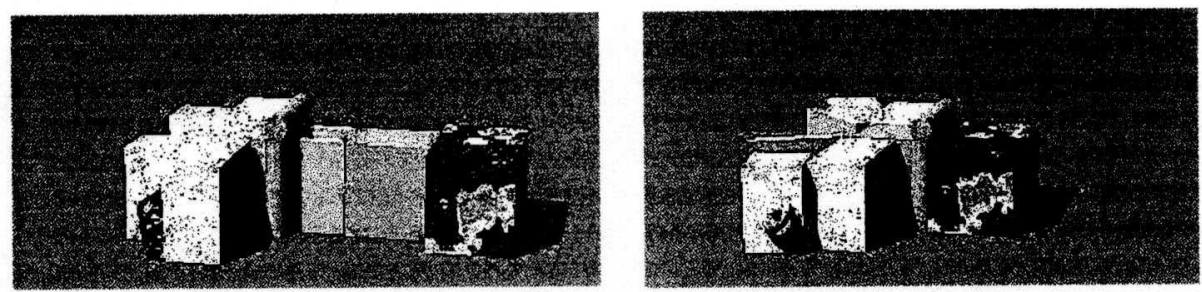

\section{LOCK:}

Figures 3.2 (a) and (b)

This model uses a displacement force mechanism to lock the fixing into the base material of the connecting structure. The fixings are attached to the back of the cladding panel (either by adhesive or screw tap), and the whole assembly aligned to pre-drilled holes in the structure. As the fixings touch the base of the hole, the locking mechanism displaces the base material and then locks into it as the above model shows.
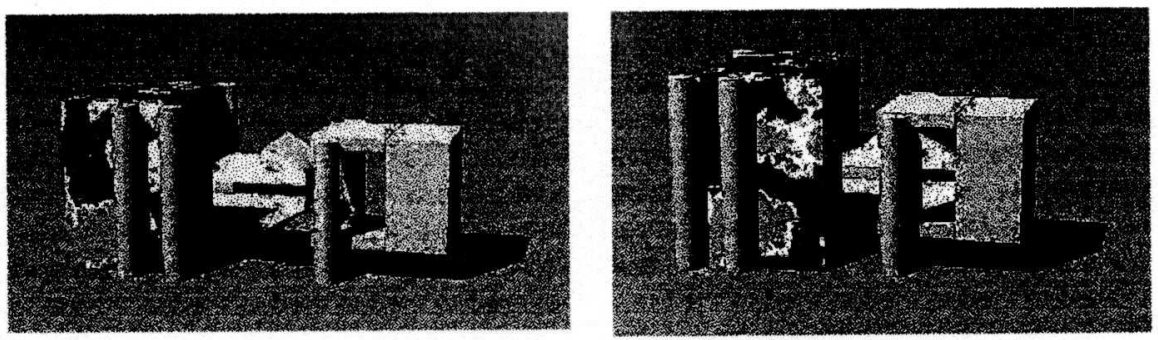

\section{SNAPFIT}

Figures 3.3 (a) and (b)

The snapfit model uses, as its name implies, a spring mechanism which displaces the fixing into its lock position. The fixings are attached to the back of the cladding panel, and the spring mechanisms lock into pre-drilled holes in the structure as shown. 

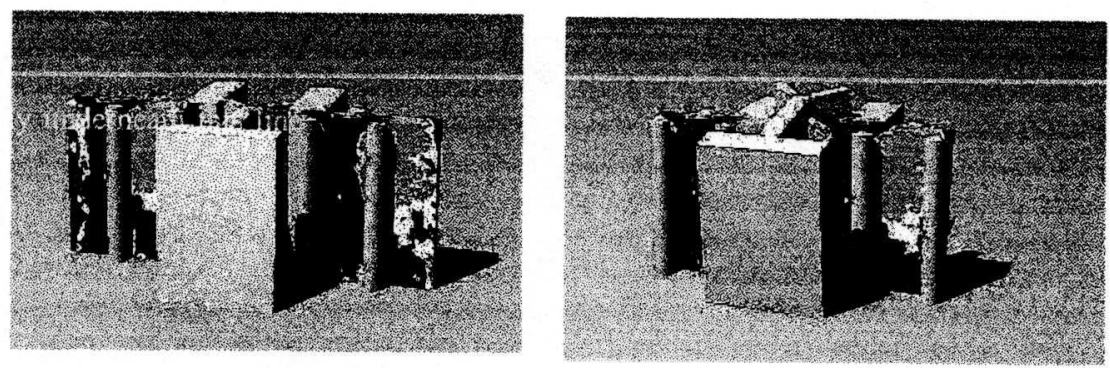

\section{CURTWALL}

Figures 3.4 (a) and (b)

Model curtwall uses the concept of curtain wall construction, whereby two cladding panels come to a joint on the structure and a ' $\mathrm{T}$ ' shape element is used to lock the two panels into position. The fixing connection at the end of the ' $\mathrm{T}$ ' section also locks into the structure behind.
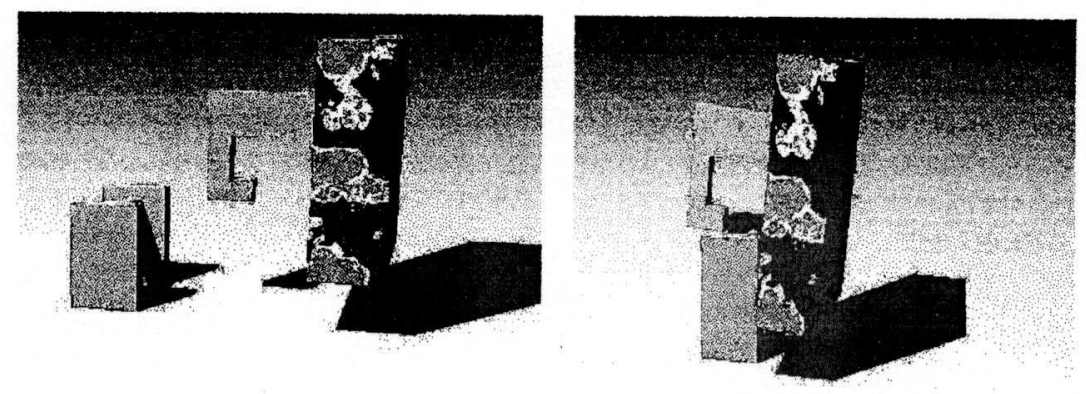

GRAVITY

Figures 3.5 (a) and (b)

The principle of the gravity model is somewhat similar to the groove, whereby a gravity arm (or hook) attached to the back of the cladding panel is aligned towards a dowel in the connecting bracket. When all hooks are aligned, the panel drops under its own weight into the bracket assembly. Once again, the weight of the panel above secures the locking mechanism.
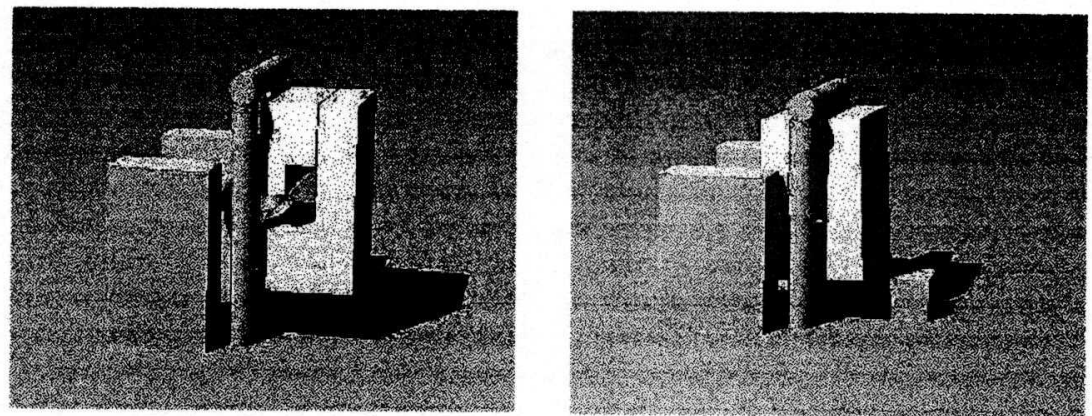

HOOK

Figures 3.6 (a) and (b)

The Hook model is based on principles similar to those of the Gravity model described above, but the locking hook is built into the cladding panel rather than attached to it. 

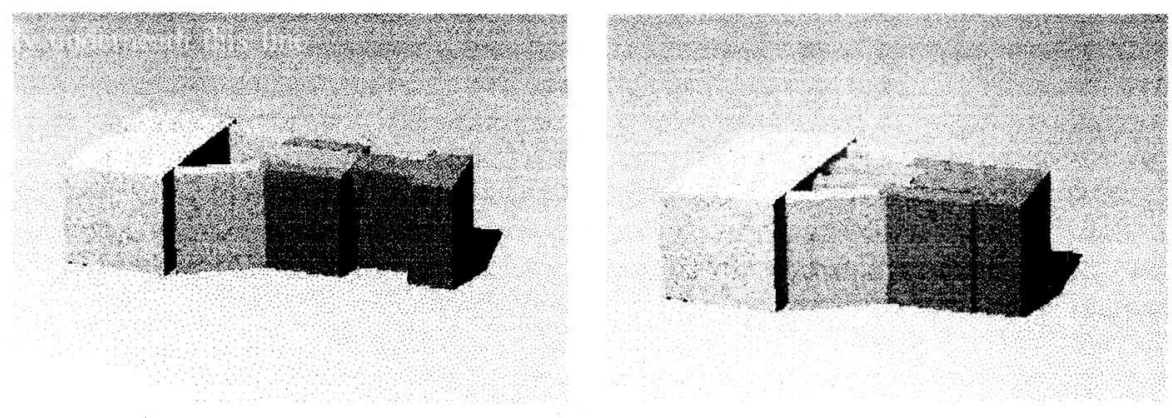

FISCH

Figures 3.7 (a) and (b)

The Fisch model is based on an blind anchorage system developed by Artur Fischer Fixing Ltd, whereby the fixing is placed into an under-reamed cavity on the unseen side the cladding and secured by application of a torque moment to the end of the fixing. This end is then bolted onto the cladding support rail.

\subsection{FURTHER DEVELOPMENT}

Further enhancements to the demonstration models are currently underway using ProEngineer and Iris Performer software to model the location, positioning and installation of typical cladding panels on frame structures. This stage forms part of the macro modelling process explained above, and includes the model of an adapted telescopic boom mobile crane. The macro models are being linked into a virtual reality image simulation, and will eventually incorporate the micro modelling of the connector details.

\subsection{CONCLUSION}

A series of new cladding to frame connectors for automated construction systems are being developed. The rational approach to these connectors is to develop and use connectors which are less labour intensive, of higher quality, are matched to the precision of the construction equipment, and which will provide other relative benefits such as improved safety on site. It is expected that the complex development and design processes for automated application of the connectors will be aided by the use of simulation software as described above.

Robust automated connector systems such as those described in this paper which consistently perform in a successful manner will be required to satisfy a growing demand in a construction industry which is moving towards comprehensive use of automation and robotics for assembly processes. 


\subsection{ACKNOWLEDGEMENT}

The work described in this paper has been carried out by the authors as part of EPSRC funded grant: GR/H 46374 "Robotic Fixing Systems for Cladding".

\section{REFERENCES}

1. Atkin B., Atkinson P., Bridgewater C., Davies B., Wing R., Assembly Techniques For Large Construction Components, Proc. 11th Int. Symp on Autmn and Rob in Constr, (ISARC), Brighton, UK, 1994, pp 473-480.

2. Atkin B., Atkinson P., Bridgewater C., Davies B., Wing R., Construction Cladding Joining Methods: A Guide to Design for Automation Assembly,: Proc. I0th Int. Symp on Autmn and Rob in Constr, (ISARC), Construction Industry Institute, Houston, Texas, USA, 1993, pp 157-164

3. Aspects of Cladding, The Institution of Structural Engineers, London, UK, August 1995.

4. Wall Cladding: Designing to Minimise defects Due to Inaccuracies and Movements, BRE Digest 223, Building Research Establishment, March 1979, Garston, UK.

5. Popo-Ola, O.S., Davidson, P.C., Sullivan, P.J., England, G.L., Steel Cladding Systems in Re/Over-clad of Buildings in: Proceedings of the International Conference on Building Envelope Systems and Technology, Singapore, Dec. 1994. pp. 51-56.

6. Keller H., Stolz H., Ziegler A., Bräunl T., Simulation and Animation of Rigid Body Systems, University of Stuttgart, IPVR, Computer Vision Group. 1993. 